\title{
Correlation Between Dynamic Changes of Chest CT Findings and Therapeutic Response of COVID-19: A Multicenter Experience in Shanghai, China
}

\author{
Jingqi Zhu ${ }^{\# 1}$, Cuisong Tang ${ }^{\# 2}$, Yuxin Shi ${ }^{3}$, Songhua Zhan ${ }^{4}$, Shuai Wang ${ }^{1}$, Litao Song ${ }^{5}$, Zhihong Shao ${ }^{6}$, \\ Guangyu Tang ${ }^{1,2}$ and Lin Zhang, ${ }^{1, *}$ \\ ${ }^{1}$ Department of Radiology, Shanghai Tenth People's Hospital, Tongii University School of Medicine, Shanghai, China \\ ${ }^{2}$ Department of Radiology, Clinical Medical College of Shanghai Tenth People's Hospital of Nanjing Medical University, Shanghai, China \\ ${ }^{3}$ Department of Radiology, Shanghai Public Health Clinical Center, Shanghai, China \\ ${ }^{4}$ Department of Radiology, Shuguang Hospital Affiliated to Shanghai University of Traditional Chinese Medicine, Shanghai, China \\ ${ }^{5}$ Department of Radiology, Shanghai Seventh People's Hospital Affiliated to Shanghai University of Traditional Chinese Medicine, Shanghai, China \\ ${ }^{6}$ Department of Radiology, Shanghai Shibei Hospital of Jing'an District, Shanghai, China \\ "Corresponding author: Department of Radiology, Shanghai Tenth People’s Hospital, Tongji University School of Medicine, Shanghai, China. Email: syfskzhanglin@163.com \\ \# These authors are contributed equally as the first author.
}

Received 2020 August 22; Revised 2021 May 23; Accepted 2021 May 25.

\section{Abstract}

Background: The novel coronavirus disease 2019 (COVID-19) has become a global public health emergency. Computed tomography (CT) offers valuable clues to the diagnosis of COVID-19. However, little is known about the correlation between dynamic changes of CT scores and therapeutic response in the course of COVID-19.

Objectives: To describe the temporal changes of CT findings and characterize the time window of disease progression on the followup CT scans of patients with COVID-19.

Patients and Methods: In this historical cohort study performed in Shanghai, China, the follow-up chest CT images of 91 patients with COVID-19 with different therapeutic responses were reviewed in multiple centers, with an emphasis on characterizing the changing trend of CT scores for lung lesions at 13 - 15 days after the symptom onset and thereafter. The CT score curve patterns were categorized into type 1 (characterized by an increase to the peak level, followed by a decrease), type 2 (characterized by a steady change without an obvious peak), and type 3 (characterized by a progressive increase).

Results: The CT scores of the progression group $(n=9)$ with a longer time to the peak were significantly higher than those of the non-progression group $(n=82)$ on the first day and days $13-15(P<0.05)$, except for the median CT scores before days $13-15$. The CT curve type 1 and type 2 were commonly observed in the non-progression group ( $63.4 \%$ and $36.6 \%$, respectively), while type 3 was more common in the progression group (88.9\%).

Conclusion: Most patients with COVID-19 show favorable responses to clinical treatments in Shanghai. Thirteen to fifteen days after the symptom onset can be considered as a turning point for the therapeutic response. The CT curve type 3 usually represents a poor response. The CT scores of patients with different therapeutic responses may overlap before days $13-15$. The changing trend of longitudinal CT scores may contribute to the prediction of disease progression.

Keywords: COVID-19, Pneumonia, Computed Tomography, Follow-up, Progression

\section{Background}

Severe acute respiratory syndrome coronavirus 2 (SARS-CoV-2), which belongs to the family Coronaviridae and the order Nidovirales, was initially reported in Wuhan, Hubei Province, China, in December, 2019. It soon became a global public health emergency, as declared by the World Health Organization (WHO) on January 30, 2020 (1). Based on reports since August 19, 2020, SARS-CoV-2 has spread rapidly around the world, accounting for 21,989,366 cases of infection (89,980 cases in China) and 775,893 deaths (4,712 cases in China with a mortality rate of $5.2 \%)$ since December 2019.

Suspected exposure, travel/residential history, initial symptoms (including fever, cough, myalgia, and fatigue), and acute pneumonia are critical to the diagnosis of COVID-19 caused by SARS-CoV-2 (2). Although the gold diagnostic standard for COVID-19 is the nucleic acid test by 
real-time reverse transcription-polymerase chain reaction (RT-PCR), chest imaging, especially computed tomography (CT), offers valuable clues to the diagnosis of COVID-19 (3). To the best of our knowledge, there have been many reports on the CT features of COVID-19 (4-10). However, there is little information about the correlation between dynamic changes of CT scores and the patients' therapeutic response in the course of COVID-19 in Shanghai, China.

\section{Objectives}

This study aimed to describe the features of chest CT scores and to characterize the time window of disease progression on follow-up CT scans in patients with COVID-19 during the progression of the disease.

\section{Patients and Methods}

\subsection{Participants}

From January 19, 2020 to March 15, 2020, a total of 91 patients diagnosed with COVID-19 were included in this study (51 patients from Shanghai Public Health Clinical Center; 17 patients from Shanghai Tenth People's Hospital affiliated to Tongji University School of Medicine; 11 patients from Shuguang Hospital affiliated to Shanghai University of Traditional Chinese Medicine; six patients from Shanghai Seventh People's Hospital affiliated to Shanghai University of Traditional Chinese Medicine; three patients from Shanghai Shibei Hospital of Jing'an District; one patient from Yueyang Hospital affiliated to Shanghai University of Traditional Chinese medicine; one patient from Putuo People's Hospital affiliated to Tongji University School of Medicine; and one patient from Shanghai Tenth People's Hospital Chongming Branch). The inclusion criteria were as follows: (1) fever or other symptoms; (2) no less than two laboratory tests performed within 12 days after the symptom onset; (3) no less than three thin-section chest CT scans within 12 days after the symptom onset; (4) the first laboratory examination and thin-section chest CT scan performed simultaneously on the first day of the patient's visit with a physician; and (5) a positive real-time RT-PCR result for SARS-CoV-2 nucleic acid in respiratory samples.

The exclusion criteria were as follows: (1) no laboratory tests performed within 13 - 15 days after the symptom onset; and (2) no thin-section chest CT scan within 13 - 15 days after the symptom onset. Clinical information, including epidemiological history, symptoms, and laboratory data, was collected in detail. All patients received treatment at Shanghai Public Health Clinical Center (a WHO-designated organization for newly emerging infectious diseases) after a definite diagnosis. This historical cohort study was approved by the institutional review boards of eight hospitals, with a waiver of written informed consent.

\subsection{CT scan Interpretation and Evaluation}

Thin-section chest CT scans were obtained without a contrast agent, with the patient in the supine position at the end of inspiration. The CT scanners included the Definition Flash (Siemens Medical Solutions, Erlangen, Germany), LightSpeed VCT (GE Medical systems, Milwaukee, USA), Ingenuity CT (Philips Medical Systems, Eindhoven, the Netherlands), and UCT 760 (United Imaging, Shanghai, China). The acquisition parameters were as follows: tube voltage, $120 \mathrm{kVp}$; tube current, 150 - $200 \mathrm{mAs}$; pitch, 1.0 - 1.5; collimation, $0.625 \mathrm{~mm}$; matrix, $512 \times 512$; and section thickness, $1-5 \mathrm{~mm}$. The images were displayed at lung (window width, 1,200 to $1,500 \mathrm{HU}$; window level, -600 to $-700 \mathrm{HU}$ ) and mediastinal (window width, $350 \mathrm{HU}$; window level, 40 $\mathrm{HU}$ ) window levels. All CT scans were reconstructed using a sharp reconstruction algorithm with a section thickness of $1-1.25 \mathrm{~mm}$.

The five predominant patterns of lung opacity on CT scans were categorized as follows: pure ground-glass opacification (GGO), GGO with interstitial opacity, GGO with consolidation, pure consolidation, and reticulation. Each lung lobe (five lung lobes per patient) was assigned a score, based on the lung volume involved $(0,0 \%$ involvement; 1, 1\% - 25\% involvement; 2, 26\% - 50\% involvement; 3, 51\% - 75\% involvement; and 4, 76\% - 100\% involvement). The total CT score was the sum of five lobar scores, ranging from 0 (no involvement) to 20 (maximum involvement) (4).

All patients underwent follow-up chest CT scans during hospitalization. The follow-up CT scans were reviewed to draw a CT score curve for assessing the progress of CT findings over time. Time-to-peak (TTP) was defined as the interval from the onset of symptoms to the peak of the CT score curve. All CT scans were interpreted and evaluated independently by two experienced radiologists (Jingqi Zhu and Guangyu Tang with 15 and 36 years of experience in thoracic radiology, respectively), who reached a consensus.

\subsection{Definition of clinical progress}

According to the guidelines of COVID-19 diagnosis and treatment proposed by China National Health Commission (trial version 7) (11), we divided the patients into 
two groups, based on their therapeutic response: nonprogression group (mild/common patients with symptoms continually improving) and progression group (severe/critical patients with aggravated symptoms, who required high flow oxygen therapy or mechanical ventilation because the ratio of partial pressure of oxygen to fraction of inspired oxygen was $\leq 300 \mathrm{mmHg}$ ).

\subsection{Statistical Analysis}

For the longitudinal evaluation of follow-up chest CT features, the study period was categorized according to the interval between the date of symptom onset and the date of the last chest CT scan during hospitalization (range: 18 - 21 days); CT was performed within three-day intervals after the onset of symptoms. Analyses were performed in PASW Statistics 25.0 (IBM, USA). Categorical variables were described as frequency rates and percentages (\%) and compared between the two groups using chi-square test or Fisher's exact test. Also, quantitative variables were first examined for normality using Shapiro-Wilk test. Normally distributed data were presented as mean \pm standard deviation (SD) and analyzed by independent samples $t$-test; otherwise, data without a normal distribution were presented as median (interquartile range [IQR]) and analyzed by Mann-Whitney U-test. Spearman's correlation test was also used to assess the relationships between the CT scores and laboratory parameters. A P-value less than 0.05 was considered to be statistically significant.

\section{Results}

\subsection{Clinical Characteristics}

In this study, 59 males and 32 females were included (mean age: $52.7 \pm 15.0$ years; range: 28 - 81; 41 cases < 50 years [45.1\%]; 50 cases $\geq 50$ years [54.9\%]). Forty-eight patients (48/91, 52.7\%) travelled to/or lived in the cities of Hubei Province, 10 (10/91, 11.0\%) patients were in contact with confirmed cases of COVID-19, and 33 (33/91, 36.3\%) patients had no definite history of contact with Hubei people or confirmed cases. The symptoms of the patients included fever (80/91, 87.9\%), upper respiratory tract symptoms (64/91, 70.3\%), fatigue (16/91, 17.6\%), myalgia (15/91, $16.5 \%)$, headache $(6 / 91,6.6 \%)$, dyspnea (4/91, 4.4\%), abdominal distention (2/91, 2.2\%), and diarrhea (2/91, 2.2\%) before hospitalization. The median time from the onset of symptoms until a doctor's visit was three days (IQR: 1 - 7; range: 1 - 9 days). The underlying diseases (18/91,19.8\%) are listed in Table 1.

\begin{tabular}{|c|c|}
\hline Information & Values $^{\mathbf{a}}$ \\
\hline \multicolumn{2}{|l|}{ Hubei Province contact history } \\
\hline Present & $48(52.7)$ \\
\hline Absent & $43(47.3)$ \\
\hline Age, $y$ & $52.7 \pm 15.0(28-81)$ \\
\hline$<50$ & $41(45.1)$ \\
\hline$\geq 50$ & $50(54.9)$ \\
\hline \multicolumn{2}{|l|}{ Sex } \\
\hline Male & $59(64.8)$ \\
\hline Female & $32(35.2)$ \\
\hline \multicolumn{2}{|l|}{ Symptoms } \\
\hline Fever & $80(87.9)$ \\
\hline Upper respiratory tract symptoms & $64(70.3)$ \\
\hline Fatigue & $16(17.6)$ \\
\hline Myalgia & $15(16.5)$ \\
\hline Headache & $6(6.6)$ \\
\hline Dyspnea & $4(4.4)$ \\
\hline Abdominal distention & $2(2.2)$ \\
\hline Diarrhea & $2(2.2)$ \\
\hline Underlying disease & $18(19.8)$ \\
\hline Hypertension & $6(6.6)$ \\
\hline Diabetes mellitus & $3(3.3)$ \\
\hline Heart disease & $2(2.2)$ \\
\hline Chronic liver disease & $2(2.2)$ \\
\hline Chronic obstructive pulmonary disease & $2(2.2)$ \\
\hline Multiple diseases & $3(3.3)$ \\
\hline
\end{tabular}

${ }^{\mathrm{a}}$ Values are expressed as No. (\%) or mean $\pm \mathrm{SD}$ (range).

In terms of response to clinical treatment, 82 patients (51 males and 31 females; mean age: $51.0 \pm 14.1$ years [range: 28 - 78 years]; 40 cases $<50$ years [ $48.8 \%$ ]; 42 cases $\geq 50$ years [51.2\%]) were assigned to the non-progression group, whereas the other nine patients ( 8 males and 1 female; mean age: $68.0 \pm 14.9$ years, range, 30 -81 years; one case $<50$ years [11.1\%] and eight cases $\geq 50$ years [88.9\%]) were assigned to the progression group. There was a significant difference between the two groups regarding age $(\mathrm{P}=$ 0.001), while there was no significant difference regarding the percentage of patients older than 50 years $(\mathrm{P}=0.071)$. All patients in the progression group were admitted to the Intensive Care Unit (ICU) to receive high flow oxygen therapy (one severe case) or mechanical ventilation (eight critical cases) at 13 - 15 days (mean: $13.9 \pm 0.8$ days) after the 
symptom onset (days 13 - 15). Therefore, days 13 - 15 after the symptom onset were considered as the watershed for therapeutic response.

\subsection{Laboratory Examinations}

Laboratory examinations for inflammatory markers were performed on the first day during the physician visit. The interval of follow-up examinations ranged from one to four days. The results of laboratory tests on the first day and days 13 - 15 are listed in Table 2. In the initial laboratory tests, the patients in the progression group had higher C-reactive protein (CRP) levels and lower eosinophil ratios compared to those in the non-progression group ( $\mathrm{P}$ $=0.010$ and $\mathrm{P}=0.030$, respectively). On days $13-15$, the progression group showed higher CRP levels, white blood cell count, neutrophil count, and neutrophil ratio $(\mathrm{P}<0.001$, $\mathrm{P}<0.001, \mathrm{P}<0.001$, and $\mathrm{P}=0.002$, respectively), despite a lower lymphocyte ratio, eosinophil ratio, and monocyte ratio, compared to the non-progression group $(\mathrm{P}=0.002$, $\mathrm{P}=0.037$, and $\mathrm{P}=0.025$, respectively).

\subsection{Longitudinal Assessment of CT Findings}

All included patients were scanned no less than four times in the follow-up chest CT examinations (425 scans; mean: $4.7 \pm 1.0$ scans; range: 4 - 7 scans) in the course of disease and no less than two follow-up chest CT examinations before days 13 - 15. The initial CT scans were acquired simultaneously with laboratory tests on the first day when the patient visited a physician. One patient in the non-progression group showed no abnormalities in the initial chest CT scans, while lung opacities appeared on the follow-up CT scans. In contrast, another patient in the non-progression group showed lung opacities in the initial chest CT scans; however, the signs disappeared on days 13-15.

The lesion distribution and CT scores on the initial day and days 13 - 15 were compared between the nonprogression and progression groups, as shown in Table 3. Comparison of the trend of changes in the longitudinal characteristics of CT scores on serial follow-up CT scans between the two groups is presented in Table 4. Differences in the occurrence of multiple lobes and bilateral involvement were not significant between the two groups, both on the initial day and days $13-15$. The CT scores on the initial day and days 13 - 15 after the symptom onset were both significantly higher in the progression group compared to the non-progression group $(\mathrm{P}=0.025$ and $\mathrm{P}<0.001$, respectively), while the median CT scores (defined as the median of CT scores obtained on the follow-up CT before days 13 -
15) increased in the progression group; however, the difference was not statistically significant $(P=0.067)$ (Figure 1 ).

Based on the follow-up CT scores from the initial day until days 13 - 15 after the symptom onset, three CT score curve patterns were categorized: type $1, \mathrm{CT}$ scores increasing to the peak level, followed by a decrease, with maximum CT score difference $>50 \%$; type 2, CT scores showing a steady change without an obvious peak for the CT scores < 50\%; and type 3, CT scores increasing progressively (Figure 2).

Type $1(63.4 \%, 52 / 82)$ and type $2(36.6 \%, 30 / 82)$ CT score curves were the most common in the non-progression group, while the CT score curve was type $1(11.1 \%, 1 / 9)$ and type $3(88.9 \%, 8 / 9)$ in the progression group, respectively, which is significantly different from the non-progression group (type $1+$ type 2 vs. type $3 ; \mathrm{P}<0.001$ ). The TTP in the non-progression group was significantly lower than the progression group $(\mathrm{P}=0.006)$.

\subsection{Relationships Between CT Scores and Laboratory Results}

The correlation analysis showed that the initial CT score was positively correlated with the initial CRP level ( $\mathrm{r}$ $=0.314, \mathrm{P}=0.048)$. The $\mathrm{CT}$ score on days $13-15$ was posi tively correlated with the CRP level and neutrophil ratio at the corresponding time point $(\mathrm{r}=0.316, \mathrm{P}=0.047$ and $\mathrm{r}=$ $0.425, \mathrm{P}=0.006$, respectively), while it was negatively correlated with the lymphocyte count and lymphocyte ratio ( $\mathrm{r}$ $=-0.375, \mathrm{P}=0.017$ and $\mathrm{r}=-0.456, \mathrm{P}=0.003$, respectively). $\mathrm{A}$ positive correlation was found between day $13-15 \mathrm{CT}$ score and TTP $(r=0.602, P<0.001)$, while the relationships between the initial CT score and TTP and between the median CT score and TTP were not significant $(r=-0.026, P=0.875$ and $\mathrm{r}=0.134, \mathrm{P}=0.411$, respectively) (Table 5).

\section{Discussion}

SARS-CoV-2 is believed to have a zoonotic origin with person-to-person transmission, which is similar to severe acute respiratory syndrome coronavirus (SARS-CoV) and the Middle East respiratory syndrome coronavirus (MERS-CoV) (12). The outbreak of COVID-19 was first reported in Wuhan, China, accounting for 3.5\% of mortality in the world $(775,893 / 21,989,366)$, which is significantly lower than that of SARS $(9.6 \%, 774 / 8098)$ and MERS (34.4\%, $858 / 2494)(13,14)$. However, COVID-19 is a highly infectious disease with non-specific symptoms in the prodromal phase. Overall, $52.7 \%$ of our patients (48/91) had a history of contact with a confirmed patient or a travel/residential 


\begin{tabular}{|c|c|c|c|c|c|c|}
\hline & \multicolumn{2}{|c|}{ Non-progression group $(\mathbf{n}=\mathbf{8 2})$} & \multicolumn{2}{|c|}{ Progression group $(\mathbf{n}=9)$} & \multirow{2}{*}{$\mathbf{P}^{\&}$} & \multirow{2}{*}{$\mathbf{P}^{\dagger}$} \\
\hline & Baseline $^{*}$ & Days $13-15^{\#}$ & Baseline & Days 13 - 15 & & \\
\hline C-reactive protein, $\mathrm{mg} / \mathrm{L}$ & $8.13(1.40-27.50)$ & $2.64(0.50-9.60)$ & $65.94(45.46-87.36)$ & $97.20(46.97-131.88)$ & 0.001 & $<0.001$ \\
\hline White blood cell count, $\times 10^{9} / \mathrm{L}$ & $5.06(4.11-5.99)$ & $4.98(4.29-5.76)$ & $6.19(3.95-11.58)$ & $10.37(8.30-15.60)$ & 0.528 & $<0.001$ \\
\hline Neutrophil count, $\times 10^{9} / \mathrm{L}$ & $2.90(2.38-3.79)$ & $2.87(2.35-3.69)$ & $4.18(3.39-5.88)$ & $8.30(6.48-14.69)$ & 0.074 & $<0.001$ \\
\hline Neutrophil ratio, \% & $62.35(54.70-70.55)$ & $57.35(49.60-67.33)$ & $82.15(45.30-89.38)$ & $84.00(73.55-94.30)$ & 0.161 & 0.002 \\
\hline Lymphocyte count, $\times 10^{9} / \mathrm{L}$ & $1.27(0.91-1.68)$ & $1.52(1.16-1.89)$ & $0.44(0.28-6.07)$ & $0.63(0.42-2.19)$ & 0.112 & 0.123 \\
\hline Lymphocyte ratio,\% & $26.10(20.73-33.15)$ & $32.95(21.05-38.60)$ & $8.15(5.75-49.03)$ & $7.40(2.93-21.25)$ & 0.135 & 0.002 \\
\hline Eosinophil count, $\times 10^{9} / \mathrm{L}$ & $0.01(0.00-0.02)$ & $0.07(0.01-0.12)$ & $0.00(0.00-0.01)$ & $0.00(0.00-0.10)$ & 0.074 & 0.101 \\
\hline Eosinophil ratio, \% & $0.20(0.00-0.58)$ & $1.35(0.20-2.35)$ & $0.00(0.00-0.08)$ & $0.00(0.00-1.28)$ & 0.032 & 0.037 \\
\hline Monocyte ratio, \% & $9.65(7.05-12.85)$ & $8.30(6.73-10.10)$ & $5.25(3.23-11.40)$ & $4.60(1.83-7.98)$ & 0.112 & 0.025 \\
\hline
\end{tabular}

${ }^{\mathrm{a}}$ Values are expressed as median (IQR).

b*, The first day when the patient visited a doctor; \#, 13 - 15 days after the symptom onset; \&, Non-progression group vs. progression group on the first day; $\dagger$, nonprogression group vs. progression group on days $13-15$.

\begin{tabular}{|c|c|c|c|c|c|c|}
\hline & \multicolumn{2}{|c|}{ Non-progression group } & \multicolumn{2}{|c|}{ Progression group } & \multirow{2}{*}{$\mathbf{P}^{\&}$} & \multirow{2}{*}{$\mathbf{P}^{\dagger}$} \\
\hline & Initial CT $^{*}\left(\mathrm{n}=\mathrm{81}^{\mathrm{B}}\right)$ & Day $13-15 C T \#\left(n=81^{C}\right)$ & Initial CT $(\mathbf{n}=9)$ & Day 13 - 15 CT $(n=9)$ & & \\
\hline Multiple lobes & $71.6 \%(58 / 81)$ & $91.4 \%(74 / 81)$ & $100 \%(9 / 9)$ & $100 \%(9 / 9)$ & 0.105 & 1.000 \\
\hline Bilateral involvement & $65.4 \%(53 / 81)$ & $82.7 \%(67 / 81)$ & $100 \%(9 / 9)$ & $100 \%(9 / 9)$ & 0.052 & 0.345 \\
\hline CT score & $3.50(1.25-5.75)$ & $6.00(4.00-7.00)$ & $6.00(5.25-6.75)$ & $14.50(13.25-15.00)$ & 0.025 & $<0.001$ \\
\hline
\end{tabular}

\begin{tabular}{|c|c|c|c|c|c|}
\hline & \multirow{2}{*}{ Median CT score ${ }^{*}$} & \multirow{2}{*}{ Time to peak ${ }^{\#}, \mathrm{~d}$} & \multicolumn{3}{|c|}{ CT score curve } \\
\hline & & & Type 1 & Type 2 & Type 3 \\
\hline Non-progression group $(n=82)$ & $5.00(3.63-7.00)$ & $8.50(6.00-11.75)$ & $63.4 \%(52 / 82)$ & $36.6 \%(30 / 82)$ & $0.0 \%(0 / 82)$ \\
\hline Progression group $(\mathbf{n}=9)$ & $7.00(6.00-8.75)$ & $16.00(13.50-17.75)$ & $11.1 \%(1 / 9)$ & $0.0 \%(0 / 9)$ & $88.9 \%(8 / 9)$ \\
\hline $\mathbf{P}^{\dagger}$ & & 0.006 & & & \\
\hline $\mathbf{P}^{\ddagger}$ & & & $<0.001$ & & \\
\hline
\end{tabular}

${ }^{\mathrm{a}}$ Values are expressed as median (IQR) or \% (number/total).

$\mathrm{b}_{*}$, The median of CT scores obtained in the follow-up CT scans before days 13 - 15 following the symptom onset; \#, the interval from the onset of symptoms until the peak of the CT score curve; \&, comparison of the median CT score between the non-progression and progression groups; $\dagger$, comparison of time to peak between the non-progression and progression groups; $\ddagger$, comparison of the type of CT score curve between the non-progression and progression groups (type 1 and 2 are combined into a group versus type 3, based on Fisher's exact test). 

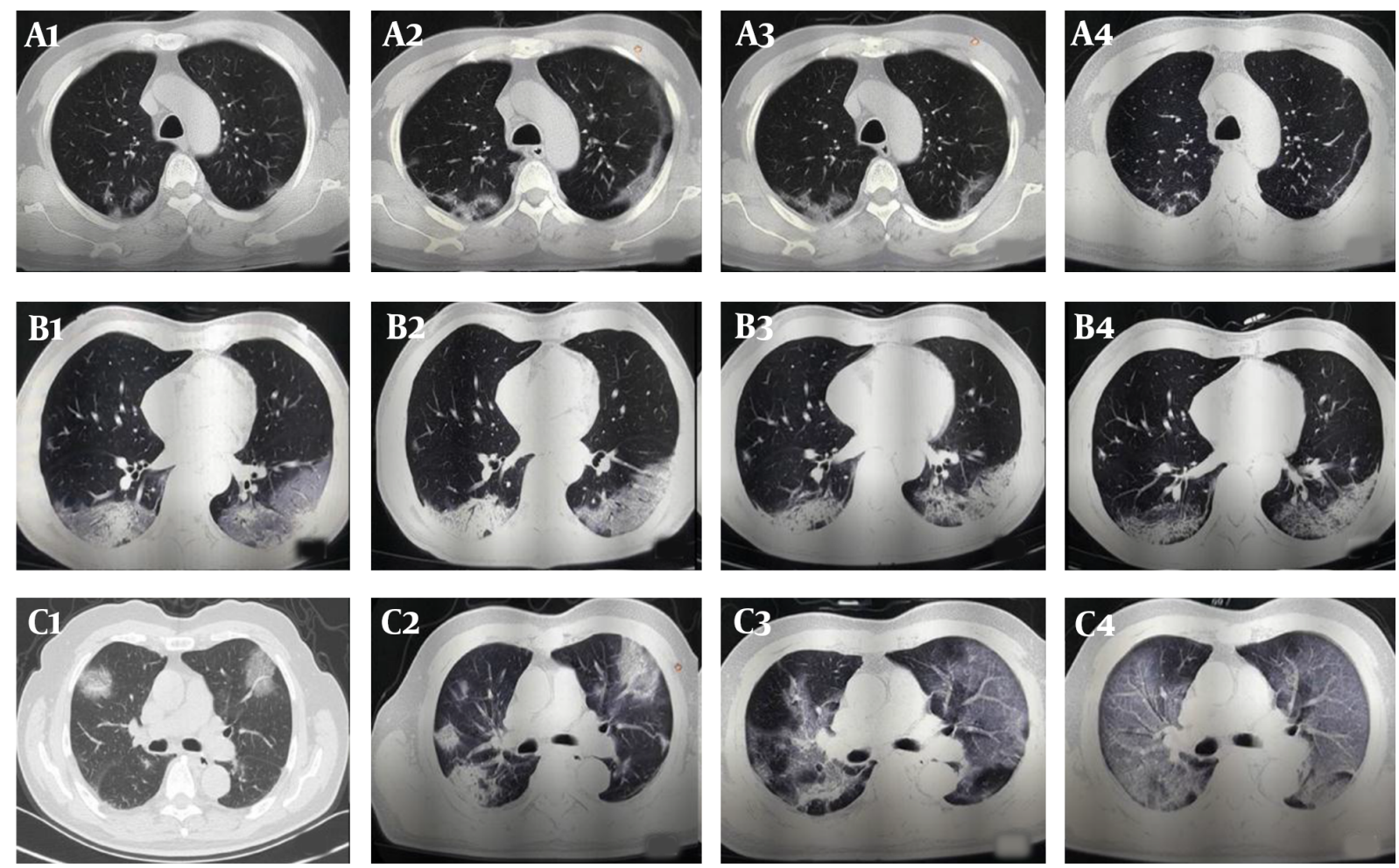

Figure 1. The follow-up CT scans of COVID-19 patients with different clinical outcomes. A, A 57-year-old male patient with good outcomes. The follow-up CT scans show that the extent of lung lesions increased from the third day after the onset of symptoms (day 3; A1) until day 9 (A2), followed by a continuous improvement on day 13 (A3) and day 21 (A4); B, A 57-year-old man with good outcomes. The follow-up CT scans show no obvious change in the extent of lung lesions on the second day after the onset of symptoms (day 2; B1), on day 8 (B2), on day 15 (B3), and on day 18 (B4); C, A 71-year-old male patient with poor outcomes. The follow-up CT scans show the extent of lung lesions continuously increasing on the second day after the onset of symptoms (day 2, C1; day 7, C2; day 13, C3; and day 18, C4).

A

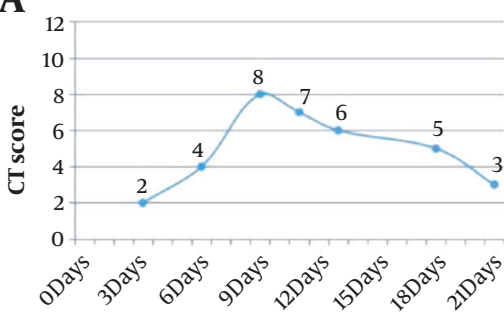

Days after onset of symptoms
B

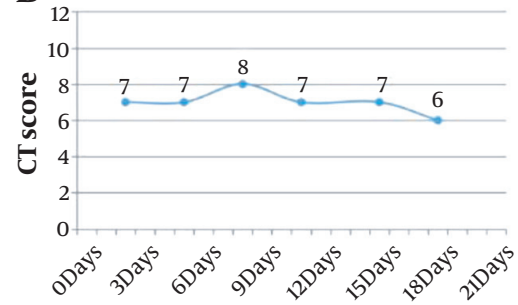

Days after onset of symptoms

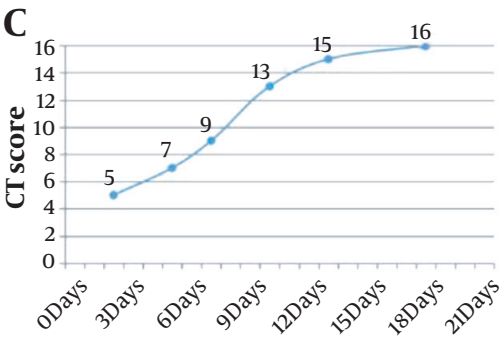

Days after onset of symptoms

Figure 2. Three types of CT score curve in COVID-19 patients. A, Type-1 CT score curve of the same patient in Figure 1A; B, Type-2 CT score curve of the same patient in Figure 1B; C, Type-3 CT score curve of the same patient in Figure 1C.

history in the cities of Hubei Province; these are important epidemiological clues to the diagnosis of COVID-19.

Consistent with recent reports (1,2), fever (80/91, 87.9\%) and upper respiratory tract symptoms (64/91, 70.3\%) were the most common presenting symptoms in our study. Compared to common/mild cases, severe/critical patients were prone to poor outcomes and high mortality $(8,9,15)$.
Old age and complications were risk factors for a poor disease prognosis (10). In our study, although the mean age of the progression group was higher than that of the nonprogression group, there was no significant difference between the two groups regarding the percentage of patients older than 50 years, which is similar to the results of a former study conducted outside Hubei Province (9). 


\begin{tabular}{|c|c|c|c|c|c|}
\hline \multirow{2}{*}{ Initial $^{*}$} & \multicolumn{2}{|c|}{ Initial CT score } & \multirow{2}{*}{ Day $13-15^{\#}$} & \multicolumn{2}{|c|}{ Day 13 - 15 CT score } \\
\hline & $\mathbf{r}^{\&}$ & $\mathbf{P}$ & & $\mathbf{r}^{8}$ & $\mathbf{P}$ \\
\hline C-reactive protein & 0.314 & 0.048 & C-reactive protein & 0.316 & 0.047 \\
\hline White blood cell count & -0.119 & 0.464 & White blood cell count & 0.147 & 0.365 \\
\hline Neutrophil count & -0.019 & 0.910 & Neutrophil count & 0.280 & 0.080 \\
\hline Neutrophil ratio & 0.049 & 0.763 & Neutrophil ratio & 0.425 & 0.006 \\
\hline Lymphocyte count & -0.280 & 0.080 & Lymphocyte count & -0.375 & 0.017 \\
\hline Lymphocyte ratio & -0.084 & 0.604 & Lymphocyte ratio & -0.456 & 0.003 \\
\hline Eosinophil count & -0.284 & 0.076 & Eosinophil count & -0.077 & 0.635 \\
\hline Eosinophil ratio & -0.153 & 0.346 & Eosinophil ratio & -0.103 & 0.528 \\
\hline Monocyte count & -0.147 & 0.367 & Monocyte count & -0.208 & 0.198 \\
\hline Monocyte ratio & -0.023 & 0.890 & Monocyte ratio & -0.304 & 0.057 \\
\hline Time to peak & -0.026 & 0.875 & Time to peak & 0.602 & $<0.001$ \\
\hline
\end{tabular}

a *, The first day when the patient visited a doctor; \&, Spearman's correlation test; \#, 13 - 15 days after the symptom onset.

It should be noted that a 30-year-old male from Wuhan without any underlying diseases developed a severe condition in our series, while his mother (62 years) who was infected by him had a mild disease. This phenomenon may be related to a decline in SARS-CoV-2 virulence and the patient's immunity status. Although the condition varied in our severe/critical patients, the time interval between the onset of symptoms and ICU admission was 13 - 15 days, which is slightly longer than that of critical patients in a single-centered study in Wuhan (survivors: 9 [6 - 12] days and non-survivors: 11 [7 - 14] days, respectively) (15). Therefore, further evidence-based research, which can reflect the degree of lung injury and predict the disease progression before the watershed (day 13 -15), is urgently needed.

Laboratory examinations for inflammatory markers can help clinicians diagnose COVID-19 and identify the disease severity in clinical practice. Recent studies have reported that an increased level of CRP may be related to an inflammatory cytokine storm, triggered by SARS-CoV-2 invasion, which generates a series of immune responses and causes changes in the peripheral white blood cells (16). In our longitudinal study, the levels of CRP in the progression group were significantly higher than the non-progression group both on the first day and days 13 - 15 after the onset of symptoms. However, compared to the non-progression group, increased white blood cell count, neutrophil count, and neutrophil ratio, beside progressively decreasing lymphocyte, eosinophil, and monocyte ratios on days 13 - 15, were found in the progression group, suggesting that the severity of inflammatory markers reflected the severity of

\section{COVID-19 in addition to CRP.}

Chest $\mathrm{CT}$ is a vital modality for the early detection and diagnosis of COVID-19, although a normal chest CT scan does not exclude the diagnosis (3). In our study, only one confirmed patient had negative CT findings at two days after the fever onset, followed by bilateral involvement after four days. Also, one patient with one lobe affected on the initial CT had a negative CT before days 13 - 15. According to previous studies, multiple lobe involvement ( $81 \%$ - 92\%) and bilateral involvement $(75 \%-86 \%)$ were the radiological features of CT scans of COVID-19 patients $(4,5,8,9)$.

Li et al. (8) found that the number of lung lobes involved in the severe/critical group was significantly higher than the common group, while no significant difference was observed in the bilateral involvement between the two groups. In our study, the occurrence of multiple lobe involvement (initial day: 67/90, 74.4\%; days 13 - 15: 83/90, 92.2\%) and bilateral involvement (initial day: 62/90, 68.9\%; days 13 -15: 76/90, 84.4\%) on CT scans was comparable to previous reports $(4,5,8,9)$. In the progression group, all patients showed multiple lobe involvement and bilateral involvement on CT scans from the initial day until days 13 - 15; this may imply a characteristic of decentralized distribution in the progression group, although no significant difference was found between the two groups.

The CT score system is usually used to accurately evaluate the extent of lung lesions $(17,18)$. A report from Chongqing Province, China, showed that the CT scores of severe/critical patients with COVID-19 were significantly higher than common COVID-19 patients (8). The follow-up 
CT scans were used to longitudinally study the extent of lung abnormalities in multiple time points in the course of the disease from the onset of symptoms. In this regard, Zhao et al. (9) reported that the progress trend in the follow-up CT scores might indicate a poor/fair response. However, their study did not appoint any specific time point to quantify the CT scores, which might lead to a lack of comparability between the results.

In the present study, serial chest CT scans were obtained in the acute nd convalescent periods. According to the watershed for therapeutic response (days 13 - 15), the baseline, median, and day 13 - 15 CT scores were calculated to compare differences between the two groups. Compared to the non-progression group, the CT scores of the progression group were higher in the baseline CT scans, while there was a progressive deterioration until days 13 - 15. However, the CT scores obtained on the median CT scans before days 13 - 15 were not significantly different between the two groups, although the scores numerically increased in the progression group compared to the nonprogression group.

In the present study, the CT score curve often overlapped for the two groups of patients and might not always match the clinical progression before the watershed (days 13 - 15). Therefore, the CT score before days 13 - 15 could not identify patients who would later progress into a severe/critical stage. The CT scores of the progression group on days 13-15 were significantly higher than the nonprogression group. Clearly, the follow-up CT scores can effectively evaluate and predict the progression of disease in a time window of 13 - 15 days after the onset of symptoms.

In addition to longitudinal CT scores, three types of CT score curve and TTP were determined based on the followup CT scans, which showed a significant difference between the non-progression and progression groups. Major patterns in the non-progression group were type 1 and type 2 , with a median TTP of 8.5 days; in other words, rapid deterioration, followed by a continuous improvement or a stable state before days 13 - 15, was a characteristic of mild/common COVID-19. On the contrary, the main pattern in the progression group was type 3 , with a median TTP of 16 days; this indicates the characteristic continuous deterioration before days 13 - 15 and thereafter in severe/critical patients.

In the present study, there was one severe patient in the progression group with a type-1 CT score curve, whose peak CT score was obtained on day 11 , followed by a gradual decline on day 15 and then a continuous increase. Continuous improvement on the CT score curve after days 13 - 15 may be critical for distinguishing mild/common cases from severe/critical cases. Combined with laboratory examinations, our study showed that lung lesions gradually aggravated to a severe/critical stage, and inflammatory markers gradually deteriorated in the progression group. The correlations between CT findings and inflammatory markers also showed that CT scores and TTP were closely related to inflammatory markers over time. Therefore, a comprehensive understanding of the trend of CT scores and inflammatory markers for 13 - 15 days after the onset of symptoms may help clinicians and radiologists to predict the therapeutic response of patients with COVID-19.

This study had several limitations. First, the data of the two groups were imbalanced, and the sample size of the progression group was relatively small. The total number of severe/critical patients with COVID-19 was no more than 20 in Shanghai. Therefore, further studies with a larger sample size, especially of severe/critical patients, are warranted. Second, some important laboratory test results, such as cytokines and immunoglobulins, were not included in our study due to incomplete data. Therefore, the relationships between the longitudinal CT scores and inflammatory factors in the serum could not be thoroughly analyzed over time.

In conclusion, most patients with COVID-19 showed favorable responses to clinical treatment in Shanghai. Thirteen to fifteen days after the onset of symptoms was considered as the watershed for therapeutic response. Type-3 CT score curve usually represents a poor response. Overall, the CT scores of patients with different therapeutic responses may overlap before the watershed (days 13 - 15). It seems that the changing trend of longitudinal CT scores combined with inflammatory markers may contribute to the prediction of COVID-19 progression.

\section{Footnotes}

Authors' Contributions: Study design: Lin Zhang, Jingqi Zhu, and Cuisong Tang. Data collection: Cuisong Tang, Yuxin Shi, Songhua Zhan, Shuai Wang, Litao Song, and Zhihong Shao. Data analysis and interpretation: Jingqi Zhu, Cuisong Tang, Shuai Wang, Guangyu Tang, and Lin Zhang. Manuscript drafting and revision: Jingqi Zhu and Guangyu Tang. All authors approved the final manuscript.

Conflict of Interests: The authors declare that they have no conflicts of interest.

Ethical Approval: Ethical approval for this study was obtained from the Ethics Committees of Shanghai Public Health Clinical Center, Shanghai Tenth People's Hos- 
pital affiliated to Tongji University School of Medicine, Shuguang Hospital affiliated to Shanghai University of Traditional Chinese Medicine, Shanghai Seventh People's Hospital affiliated to Shanghai University of Traditional Chinese Medicine, Shanghai Shibei Hospital of Jing'an District, Yueyang Hospital affiliated to Shanghai University of Traditional Chinese Medicine, Putuo People's Hospital affiliated to Tongji University School of Medicine, and Shanghai Tenth People's Hospital Chongming Branch.

Funding/Support: This work was supported by the National Natural Science Foundation of China (code: 81871325).

Informed Consent: The requirement for informed consent was waived.

\section{References}

1. Jin YH, Cai L, Cheng ZS, Cheng H, Deng T, Fan YP, et al. A rapid advice guideline for the diagnosis and treatment of 2019 novel coronavirus (2019-nCoV) infected pneumonia (standard version). Mil Med Res. 2020;7(1):4. doi: 10.1186/s40779-020-0233-6. [PubMed: 32029004]. [PubMed Central: PMC7003341].

2. Huang C, Wang Y, Li X, Ren L, Zhao J, Hu Y, et al. Clinical features of patients infected with 2019 novel coronavirus in Wuhan, China Lancet. 2020;395(10223):497-506. doi: 10.1016/S0140-6736(20)30183-5. [PubMed: 31986264]. [PubMed Central: PMC7159299].

3. Kanne JP. Chest CT Findings in 2019 Novel Coronavirus (2019-nCoV) Infections from Wuhan, China: Key Points for the Radiologist. Radiology. 2020;295(1):16-7. doi:10.1148/radiol.2020200241. [PubMed: 32017662]. [PubMed Central: PMC7233362].

4. Chung M, Bernheim A, Mei X, Zhang N, Huang M, Zeng X, et al. CT Imaging Features of 2019 Novel Coronavirus (2019-nCoV). Radiology. 2020;295(1):202-7. doi: 10.1148/radiol.2020200230. [PubMed 32017661]. [PubMed Central: PMC7194022].

5. Song F, Shi N, Shan F, Zhang Z, Shen J, Lu H, et al. Emerging 2019 Novel Coronavirus (2019-nCoV) Pneumonia. Radiology. 2020;295(1):210-7. doi: 10.1148/radiol.2020200274. [PubMed: 32027573]. [PubMed Central: PMC7233366].

6. Pan F, Ye T, Sun P, Gui S, Liang B, Li L, et al. Time Course of Lung Changes at Chest CT during Recovery from Coronavirus Disease 2019 (COVID19). Radiology. 2020;295(3):715-21. doi: 10.1148/radiol.2020200370. [PubMed: 32053470]. [PubMed Central: PMC7233367].

7. Ai T, Yang Z, Hou H, Zhan C, Chen C, Lv W, et al. Correlation of Chest CT and RT-PCR Testing for Coronavirus Disease 2019 (COVID-
19) in China: A Report of 1014 Cases. Radiology. 2020;296(2):E32-40. doi: 10.1148/radiol.2020200642. [PubMed: 32101510]. [PubMed Central: PMC7233399].

8. Li K, Wu J, Wu F, Guo D, Chen L, Fang Z, et al. The Clinical and Chest CT Features Associated With Severe and Critical COVID-19 Pneumonia. Invest Radiol. 2020;55(6):327-31. doi: 10.1097/RLI.0000000000000672. [PubMed: 32118615]. [PubMed Central: PMC7147273].

9. Zhao W, Zhong Z, Xie X, Yu Q, Liu J. CT Scans of Patients with 2019 Novel Coronavirus (COVID-19) Pneumonia. Theranostics. 2020;10(10):460613. doi: 10.7150/thno.45016. [PubMed: 32292517]. [PubMed Central: PMC7150491].

10. Shi H, Han X, Jiang N, Cao Y, Alwalid O, Gu J, et al. Radiological findings from 81 patients with COVID-19 pneumonia in Wuhan, China: a descriptive study. Lancet Infect Dis. 2020;20(4):425-34. doi: 10.1016/S1473-3099(20)30086-4. [PubMed: 32105637]. [PubMed Central: PMC7159053]

11. China National Health Commission. Diagnosis and treatment of pneumonitis caused by new coronavirus (trial version 7). 2020, [cited 20 Aug 2020]. Available from: http://www.nhc.gov.cn/yzygj/s7653p/ 202003/46c9294a7dfe4cef80dc7f5912eb1989.shtml.

12. Habibzadeh P, Stoneman EK. The Novel Coronavirus: A Bird's Eye View. Int J Occup Environ Med. 2020;11(2):65-71. doi: 10.15171/ijoem.2020.1921. [PubMed: 32020915]. [PubMed Central: PMC7205509].

13. World Health Organization. Summary of probable SARS cases with onset ofillness. 2003, [cited 20 Aug 2020]. Available from: https://www.who. int/csr/sars/country/table2004_04_21/en/.

14. World Health Organization. Middle East respiratory syndrome Coronavirus (MERS-CoV). 2019, [cited 20 Aug 2020]. Available from: https: //www.who.int/emergencies/mers-cov/en/.

15. Yang X, Yu Y, Xu J, Shu H, Xia J, Liu H, et al. Clinical course and outcomes of critically ill patients with SARS-CoV-2 pneumonia in Wuhan, China: a single-centered, retrospective, observational study. Lancet Respir Med. 2020;8(5):475-81. doi: 10.1016/S2213-2600(20)30079-5. [PubMed: 32105632]. [PubMed Central: PMC7102538].

16. Chen N, Zhou M, Dong X, Qu J, Gong F, Han Y, et al. Epidemiological and clinical characteristics of 99 cases of 2019 novel coronavirus pneumonia in Wuhan, China: a descriptive study. Lancet. 2020;395(10223):507-13. doi: 10.1016/S0140-6736(20)30211-7. [PubMed: 32007143]. [PubMed Central: PMC7135076].

17. Chang YC, Yu CJ, Chang SC, Galvin JR, Liu HM, Hsiao $\mathrm{CH}$, et al. Pulmonary sequelae in convalescent patients after severe acute respiratory syndrome: evaluation with thin-section CT. Radiology. 2005;236(3):1067-75. doi: 10.1148/radiol.2363040958. [PubMed: 16055695].

18. Ooi GC, Khong PL, Muller NL, Yiu WC, Zhou LJ, Ho JC, et al. Severe acute respiratory syndrome: temporal lung changes at thin-section CT in 30 patients. Radiology. 2004;230(3):836-44. doi: 10.1148/radiol.2303030853. [PubMed: 14990845]. 